\title{
Clinical Usefulness of Preoperative and Postoperative Chest Computed Tomography for Colorectal Cancer
}

\author{
Jeong Hui Lee, Byung Kyu Ahn, Young Soo Nam, Kang Hong Lee \\ Department of Surgery, Hanyang University College of Medicine, Seoul, Korea
}

Purpose: This research sought to identify the utility value of chest computed tomography (CT) when it comes to the diagnosis of lung metastasis in cases of colorectal cancer.

Methods: From September 2004 to January 2008, 266 patients who were treated for colorectal cancer at Department of Surgery, Hanyang University College of Medicine, were divided into two groups: one that underwent preoperative and postoperative periodical chest CT (periodical inspection group, PIG; May 2006 to January 2008, 135 patients) and one that did not undergo periodical chest CT (non-periodical inspection group, NPIG; September 2004 to April 2006, 131 patients) for comparison.

Results: The overall lung metastasis diagnosis rates did not manifest any significant difference. The times to diagnose lung metastasis patients were 6.3 months and 15.7 months for the PIG and the NPIG, respectively $(\mathrm{P}=0.022)$. The size of the metastatic lung nodule was smaller in the PIG than in the NPIG $(<1 \mathrm{~cm}$ in $9 / 9$ patients vs. $<1 \mathrm{~cm}$ in $6 / 9$ patients in the PIG and the NPIG, respectively; $\mathrm{P}=0.02)$. A solitary lung metastasis was more frequently found in the PIG (5/9 patients) than in the NPIG (1/11 patients) $(\mathrm{P}=0.024)$. During the follow-up period, $100 \%(2 / 2$ patients $)$ and $60 \%(3 / 5$ patients $)$ of the patients in the PIG and the NPIG, respectively, with stage III cancer underwent a lung metastasectomy $(\mathrm{P}=0.002)$. Conclusion: Chest CT enables early diagnosis with a smaller size and a lower number of lung metastases in patients with colorectal cancer. Moreover, pulmonary the rate of the pulmonary resection for selected patients may be improved. However, the contribution of chest CT to increasing the survival rate must be investigated in a prospective randomized study.

\section{Keywords: Chest CT; Colorectal cancer; Lung metastasis}

\section{INTRODUCTION}

Recently, colorectal cancer has been rapidly increasing in Korea, and its incidence is the 4th highest among all cancers [1]. Even if colorectal cancer is treated with a radical resection and postsurgical chemotherapy, as well as radiation therapy,

Received: May 28, 2010 Accepted: September 20, 2010

Correspondence to: Kang Hong Lee, M.D.

Colorectal Division, Department of Surgery, Hanyang University College of Medicine, Haengdang 1-dong, Seongdong-gu, Seoul 133-791, Korea

Tel: +82-2-2290-8447, Fax: +82-2-2281-0224

E-mail: leekh@hanyang.ac.kr

This article was poster presented at the Korean Surgical Society 61st Symposium in 2009.

(C) 2010 The Korean Society of Coloproctology

This is an open-access article distributed under the terms of the Creative Commons Attribution NonCommercial License (http://creativecommons.org/licenses/by-nc/3.0) which permits unrestricted noncommercial use, distribution, and reproduction in any medium, provided the original work is properly cited. the recurrence rate is reported to be up to approximately $50 \%$, and pulmonary metastasis is the most frequent extraperitoneal organ metastasis, its frequency being approximately $10 \%$ [2-5]. In 1944, Blalock reported for the first time a lung metastasectomy for treating colorectal cancer patients with pulmonary metastasis; after which several studies reported that surgery for pulmonary metastasis improved the survival rate [6-10]. Therefore, the rapid and accurate diagnosis of pulmonary metastasis by performing surgery for pulmonary metastasis in colorectal cancer patients has been recognized as one of the most important steps for improving the survival rate.

Recently, among diverse methods applied to diagnose pulmonary metastasis of colorectal cancer, chest computed tomography (CT) was found to be effective; nonetheless, for the diagnosis of pulmonary metastasis during follow-up observation, the role of chest CT is not clear [11]. In our study, in colorectal cancer patients, the usefulness of chest CT for the diagnosis of pulmonary metastasis prior to resection and the useful- 
ness of chest CT during the follow-up observation after radical resection of colorectal cancer were examined.

\section{METHODS}

Among patients treated for colorectal cancer at our hospital from September 2004 to January 2008, the study was conducted on 266 patients who did not develop primary tumors in other organs. At our hospital, chest CT has been performed prior to surgery and after surgery as a regular test from May 2006. This study was conducted by dividing the subjects into two patient groups and then comparing the two groups. In the first group, perisurgical chest CT was performed as a regular test from May 2006 (regular test group, periodical inspection group; May 2006 to January 2008, 32.0-month average follow-up observation period, 135 patients) while in the second group, the patients were treated without regular perisurgical CT (nonregular test group, non-periodical inspection group; September 2004 to April 2006, 30.9-month average follow-up observation period, 131 patients). The regular test group, periodical inspection group, consisted of patients treated during 21 months from May 2006 to January 2008, and the non-regular test group, non-periodical inspection group, consisted of patients treated during 21 months from September 2004 to April 2006. In the periodical inspection group, the follow-up observation period of patients treated in January 2008 was 22 months. Similarly, in the non-periodical inspection group, the follow-up observation period of patients treated in April 2006 was 22 months. None of the patients in either group dropped out during the follow-up observation.

As presurgical tests, colonoscopy, biopsy, chest radiographs, carcinoembryonic antigen (CEA) testing, and abdominopelvis CT were performed. During the postsurgical follow-up period, CEA testing and chest radiography were performed at 3-month intervals, and abdominopelvis CT was performed at 6-month intervals. For the periodical inspection group, chest CT was additionally performed prior to surgery, after surgery, and at 6-month intervals during the follow-up observation period. Among patients undergoing radical surgery, considering risk factors for recurrence, stage II, III and IV patients were treated with chemotherapy alone or in combination with radiation therapy after surgery.

Pulmonary metastasis was diagnosed by using chest radiography, chest CT, positron emission tomography, and biopsy. In the initial test, small lesions or lesions with nonspecific shapes, which were difficult to diagnose as pulmonary metastasis, were diagnosed as suspicious lesions, and if the size of a lesion increased or its morphology became specific during the followup observation, it was diagnosed as pulmonary metastasis. In the non-periodical inspection group, CT was performed only for cases suspected of having pulmonary metastasis based on chest radiography for accurate diagnosis. For statistical analy- sis, the $X^{2}$ method and the $\mathrm{t}$-test were applied, and P-values less than 0.05 were determined to be significant.

\section{RESULTS}

\section{Characteristics of the subjects}

The follow-up observation periods of the two groups were 32.0 months and 30.9 months for the periodical inspection group and the non-periodical inspection group, respectively, and were not statistically different. The ratio of males and females in both groups was $1.25: 1.67$, which was not a statistically significantly difference. Concerning the site of colorectal cancer, the numbers of cases of colon cancer (59\%) and rectal cancer $(41 \%)$ in the periodical inspection group were not different from those of colon cancer (56\%) and rectal cancer (44\%) in the non-periodical inspection group. Similarly, in regard to the ratio of disease stages (American Joint Committee on Cancer classification. 6th ed.), stage I, stage II, stage III, and stage IV cancers represented $16 \%, 32 \%, 30 \%$, and $22 \%$, respectively, of the periodical inspection group and $17 \%, 27 \%, 38 \%$, and $18 \%$ of the non-periodical inspection group, the differences not being statistically significant (Table 1).

\section{Diagnosis and treatments of pulmonary metastasis}

The rate and the time of the detection of the pulmonary metastasis In the periodical inspection group, perisurgical pulmonary metastasis of colorectal cancer was detected in 9 patients (5 patients prior to surgery $[5 / 9,56 \%]$ and 4 patients during the postsurgical follow-up observation period $[4 / 9,44 \%]$ ) whereas in the non-periodical inspection group, it was detected in 11 patients ( 1 patient prior to surgery $[1 / 11,9 \%]$ and 10 patients after surgery $[10 / 11,91 \%])$; the frequencies of metastasis for the two groups were not different (Table 2). The mean period to the diagnosis of pulmonary metastasis for the periodical in-

Table 1. Clinicopathologic characteristics

\begin{tabular}{lccc}
\hline & PIG $(\mathrm{n}=135)$ & NPIG $(\mathrm{n}=131)$ & P-value \\
\hline Age (mean) & 62 & 62 & - \\
Sex & & & 0.245 \\
$\quad$ Male & $75(56)$ & $82(63)$ & \\
$\quad$ Female & $60(44)$ & $49(37)$ & \\
$\quad$ Tumor location & & & 0.562 \\
$\quad$ Colon & $80(59)$ & $73(56)$ & \\
$\quad$ Rectum & $55(41)$ & $58(44)$ & \\
Primary cancer stage ${ }^{\text {a }}$ & & & 0.504 \\
$\quad$ I : II : III : IV & $22: 43: 40: 30$ & $22: 35: 50: 24$ & \\
Follow-up duration & 32 & 30.9 & - \\
$\quad$ (mean, mo) & & & \\
\hline
\end{tabular}

Values are presented as number (\%) unless otherwise indicated.

PIG, periodical inspection group; NPIG, non-periodical inspection group.

aBy 6th edition of the American Joint Committee on Cancer classification. 
spection group was 6.3 months, which was significantly shorter than the 15.7 months for the non-periodical inspection group $(\mathrm{P}=0.022)$ (Fig. 1).

In the non-periodical inspection group, a solitary pulmonary metastasis was diagnosed in only $9.1 \%$ of the cases ( $1 / 11$ cases) while in the periodical inspection group, it was diagnosed in $55.6 \%$ of the cases (5/9 cases); the frequency of the detection of a solitary pulmonary metastasis was higher in the latter group $(\mathrm{P}=0.024)$. The lesion size was smaller than $1 \mathrm{~cm}$ in $54.5 \%$ of the non-periodical inspection group (6/11 cases) and $100 \%$ (9 cases) of the periodical inspection group. Although the sizes of the diagnosed pulmonary lesions were smaller in the periodical inspection group $(\mathrm{P}=0.02)$, the distribution of lesions in the periodical inspection group was not statistically different from that in the non-periodical inspection group $(\mathrm{P}$ $>0.05$ ) (Table 3).

The detection rate and the time of the pulmonary metastasis, as well as suspicious lesions

Twelve cases of pulmonary metastasis and suspicious lesions were detected during the postsurgical follow-up observation

Table 2. Rate of diagnosis of metastatic lesions

\begin{tabular}{lccc}
\hline & PIG & NPIG & P-value \\
\hline Time & & & \\
Preoperative & $5(5 / 135,3.7 \%)$ & $1(1 / 131,0.8 \%)$ & 0.106 \\
Follow-up & $4(4 / 130,3.1 \%)$ & $10(10 / 130,7.6 \%)$ & 0.442 \\
Total & $9(9 / 135,6.7 \%)$ & $11(11 / 131,8.2 \%)$ & 0.562 \\
\hline
\end{tabular}

PIG, periodical inspection group; NPIG, non-periodical inspection group.

Table 3. Patterns of metastatic lesions

\begin{tabular}{lccc}
\hline & PIG & NPIG & P-value \\
\hline No. of metastasis & 5 & 1 & 0.024 \\
$\quad$ Solitary & 4 & 10 & \\
$\quad$ Multiple & & & 0.199 \\
Site of metastasis & 5 & 3 & \\
$\quad$ One lung & 4 & 8 & \\
$\quad$ Both lungs & & & 0.020 \\
Size of metastasis (cm) & 9 & 6 & \\
$<1$ & 0 & 5 & \\
$\geq 1$ & 0
\end{tabular}

PIG, periodical inspection group; NPIG, non-periodical inspection group.

Table 4. Rate of diagnosis of metastatic/suspicious lesions

\begin{tabular}{lccc}
\hline & PIG & NPIG & P-value \\
\hline Time & & & \\
$\quad$ Preoperative & $5(5 / 135,3.7 \%)$ & $1(1 / 131,0.8 \%)$ & 0.106 \\
$\quad$ Follow-up & $12(12 / 130,9.2 \%)$ & $10(10 / 130,7.6 \%)$ & 0.657 \\
Total & $17(17 / 135,12.7 \%)$ & $11(11 / 131,8.2 \%)$ & 0.267 \\
\hline
\end{tabular}

PIG, periodical inspection group; NPIG, non-periodical inspection group. period in the periodical inspection group. Among them, 4 cases were diagnosed as pulmonary metastasis, and the remaining 8 cases could be diagnosed only as suspicious lesions during the study period. On the other hand, in the non-periodical inspection group, 10 cases were diagnosed as suspicious lesions by chest radiography, and all were diagnosed as pulmonary metastasis by chest CT. During the follow-up observation period, the incidences of pulmonary metastasis and suspicious lesions in the periodical inspection group and the non-periodical inspection group were not statistically different (Table 4). Nonetheless, the mean period to the detection of suspicious lesions in the periodical inspection group was 11.4 months while in the non-periodical inspection group, it was 17.3 months. Thus, the time to detection of a suspicious lesion was significantly shorter in the periodical inspection group $(\mathrm{P}=0.018)$ (Fig. 2).

The rate of the resection of the pulmonary metastasis Among 5 patients in the periodical inspection group diagnosed as having pulmonary metastasis prior to surgery, the chest radiographs of 3 patients were normal. Among those 3 patients, 2 patients had a resectable solitary metastasis nodule; thus, a radical resection of the colorectal cancer and a lung metastasectomy were performed simultaneously. In the third patient, a bilateral multicentric pulmonary metastasis was detected; thus, a lung metastasectomy was not performed. One patient in the non-periodical inspection group who had been diagnosed as having a pulmonary metastasis prior to surgery had

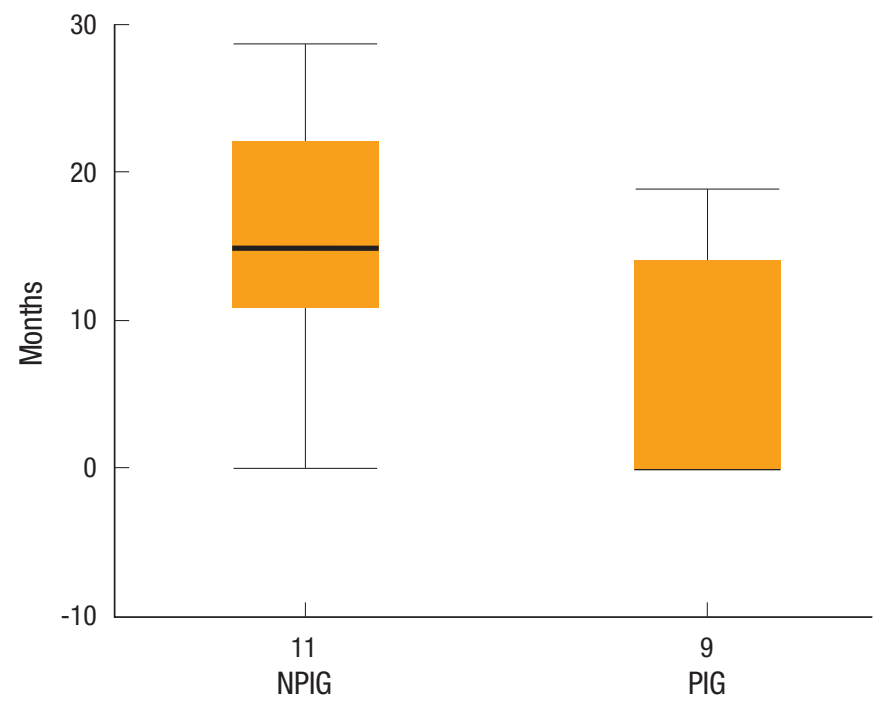

Fig. 1. Diagnosis of metastatic lesions. The lead time to diagnose lung metastasis patients was 6.3 months and 15.7 months for the periodical and the non-periodical inspection groups, respectively $(\mathrm{P}=$ $0.022)$.

NPIG, non-periodical inspection group; PIG, periodical inspection group. 


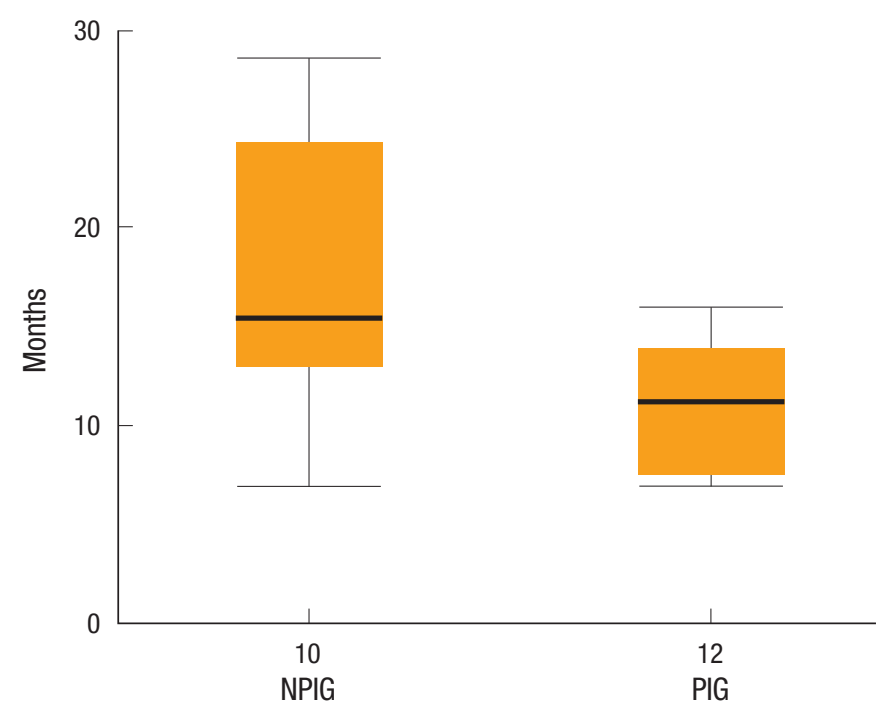

Fig. 2. Diagnosis of metastatic/suspicious lesions (follow-up period) $(\mathrm{P}=0.018)$. The lead time to confirm the presence of or the suspicion of having lung metastasis was 11.4 months and 17.3 months for the periodical and the non-periodical inspection groups $(\mathrm{P}=0.018)$. NPIG, non-periodical inspection group; PIG, periodical inspection group.

a bilateral multicentric pulmonary metastasis; thus, a lung metastasectomy was not performed.

For patients in the periodical inspection group who had a pulmonary metastasis detected during the follow-up observation period, 2 were in stage III and 2 were in stage IV; in the non-periodical inspection group, 2 were in stage II, 3 were in stage III, and 5 were in stage IV. Two patients in the periodical inspection group $(2,50 \%$, average 10.8 months) underwent a lung metastasectomy, as did three in the non-periodical inspection group (3, 30\%, average 22.0 months) (Table 5). Excluding patients on whom a lung metastasectomy could not be performed at the time of the diagnosis of pulmonary metastasis due to causes other than pulmonary metastasis (multicentric liver metastasis [2 patients in the non-periodical inspection group and 1 patient in the periodical inspection group] and peritoneal dissemination [3 patients in the non-periodical inspection group and 1 patient in the periodical inspection group]), the lung metastasectomy rate of the periodical inspection group was $100 \%$ ( $2 / 2$ cases), and that of the nonperiodical inspection group was $60 \%$ (3/5 cases), the rate of the periodical inspection group being statistically higher $(\mathrm{P}=$ 0.002) (Table 6). Of the 2 patients in the periodical inspection group whose pulmonary metastasis was detected by using chest $\mathrm{CT}$ and who thus underwent a lung metastasectomy, chest radiographs at the time of the diagnosis of pulmonary metastasis were normal.
Table 5. Stage and metastasectomy of metastatic lesions during the follow-up period

\begin{tabular}{lccccc}
\hline & \multicolumn{5}{c}{ Stage } \\
\cline { 2 - 6 } & Metastasectomy & I & II & III & IV \\
\hline PIG & $(+)$ & 0 & 0 & 2 & 0 \\
& $(-)$ & 0 & 0 & 0 & 2 \\
\multirow{2}{*}{ NPIG } & $(+)$ & 0 & 2 & 1 & 0 \\
& $(-)$ & 0 & 0 & 2 & 5 \\
\hline
\end{tabular}

PIG, periodical inspection group; NPIG, non-periodical inspection group.

Table 6. Resectability of metastatic lesions during the follow-up period

\begin{tabular}{lcc}
\hline \multirow{2}{*}{ Stage } & \multicolumn{2}{c}{ Resectability } \\
\cline { 2 - 3 } & $\mathrm{I}+\mathrm{II}+\mathrm{III}+\mathrm{IV}$ & $\mathrm{I}+\mathrm{II}+\mathrm{III}$ \\
\hline $\mathrm{PIG}$ & $50 \%(2 / 4)$ & $100 \%(2 / 2)$ \\
$\mathrm{NPIG}$ & $30 \%(3 / 10)$ & $60 \%(3 / 5)$ \\
P-value & - & 0.002 \\
\hline
\end{tabular}

PIG, periodical inspection group; NPIG, non-periodical inspection group.

\section{DISCUSSION}

In a study that prospectively compared chest $\mathrm{CT}$ and chest radiography for the diagnosis of pulmonary metastasis in solid tumors (24 cases), chest CT was shown to be useful [12]. In colorectal cancer patients, when chest $\mathrm{CT}$ and chest radiography performed prior to surgery for the diagnosis of pulmonary metastasis were compared, the positive prediction rate of chest radiography was $67 \%$, and it was found to be useful for the diagnosis of pulmonary metastasis prior to surgery [13]. Nevertheless, in a study that evaluated the usefulness of chest CT for the assessment of the presence of pulmonary metastasis prior to a lung metastasectomy in colorectal cancer patients with liver metastasis, the positive prediction rate was not significantly different from that for chest radiography; thus, its usefulness was low [14]. In our study, in colorectal cancer patients, when chest CT was applied during a follow-up observation period identical to chest radiography, the diagnosis rate of pulmonary metastasis was not increased. Nevertheless, the time to the diagnosis of pulmonary metastasis was significantly shorter, and the sizes of pulmonary metastasis lesions were small; thus, the pulmonary metastasis could be diagnosed earlier.

When colorectal cancer metastasis was not treated, the average survival period of patients was less than 10 months, but $5 \%$ of the patients survived for longer than 5 years [15]. Treatments for pulmonary metastasis of colorectal cancer are systemic chemotherapy, radio-frequency ablation and other conservative treatments, and surgical resection. A lung metastasectomy was confirmed to improve the survival rate; thus, the indications for a lung metastasectomy have recently been ex- 
panded $[5,16,17]$. If pulmonary metastasis that developed during the postsurgical follow-up observation period is detected early and resected aggressively, the survival rate is improved [7]. In our study, the rates of lung metastasectomies for patients whose pulmonary metastasis was definitely diagnosed during the follow-up observation period was $50 \%$ and $30 \%$, respectively, for the periodical inspection group and the non-periodical inspection group. The average time to the lung metastasectomy for the periodical inspection group was 10.8 months, which was shorter than the 22.0 months for the nonperiodical inspection group. In particular, considering only patients with cancer lower than stage III, the rate of lung metastasectomies for the periodical inspection group was $100 \%$, and that for the non-periodical inspection group was $60 \%$, but this difference was not statistically significant. Therefore, chest CT is thought to facilitate the early detection of pulmonary metastasis, which should be of help in finding patients with operable pulmonary metastasis.

In the periodical inspection group, at the time of definite diagnosis of pulmonary metastasis, chest radiography was nor$\mathrm{mal}$ in $66.7 \%$ of the cases (6/9 cases). On the other hand, in the non-periodical inspection group, in the 11 cases in which pulmonary metastasis suspicious lesions had been detected by chest radiography, and thus chest CT had been performed, pulmonary metastasis could be confirmed in all cases. In addition, in the periodical inspection group, among 12 cases in which pulmonary metastasis or suspicious lesions had been detected by chest CT during the follow-up observation, only 4 cases were definitely diagnosed as pulmonary metastasis. The remaining 8 cases could not be diagnosed definitely as pulmonary metastasis; thus, additional tests were required.

In summary, this study suggests that chest CT could be of help in detecting pulmonary metastasis in colorectal cancer patients early and, thus, in finding patients with operable pulmonary metastasis. Nonetheless, our study has limitations; the observation periods of the two groups were different, and the study was a retrospective one. In the future, to see whether the early diagnosis of pulmonary metastasis and a subsequent lung metastasectomy improves the survival rate, prospective studies are required.

In colorectal cancer patients, preoperative chest CT does not increase the rate of diagnosis of pulmonary metastasis. Nevertheless, in comparison with chest radiography, chest CT diagnoses pulmonary metastasis or suspicious lesions early, and when the size of the pulmonary lesion is small or the lesion is a solitary lesion, the lung metastasectomy rate tends to be increased. However, to see whether detecting pulmonary metastasis early by chest tomography and performing a lung metastasectomy improves the survival rate of patients, additional studies are required.

\section{ACKNOWLEDGMENT}

This work was supported by Hanyang University (2008-8771).

\section{CONFLICT OF INTEREST}

No potential conflict of interest relevant to this article was reported.

\section{REFERENCES}

1. Korean central cancer registry center, Ministry of health \& welfare. Annual report of Korean central cancer registry (2002.12002.12). Goyang: Korean Central Cancer Registry Center, Ministry of Health \& Welfare; 2003.

2. Vogelsang H, Haas S, Hierholzer C, Berger U, Siewert JR, Prauer $\mathrm{H}$. Factors influencing survival after resection of pulmonary metastases from colorectal cancer. Br J Surg 2004;91:1066-71.

3. Zink S, Kayser G, Gabius HJ, Kayser K. Survival, disease-free interval, and associated tumor features in patients with colon/rectal carcinomas and their resected intra-pulmonary metastases. Eur J Cardiothorac Surg 2001;19:908-13.

4. Kim PS, Moon SM, Hwang DY. Lung metastasis of colorectal cancer. J Korean Soc Coloproctol 2006;22:380-86.

5. Lee YC, Kim NK, Baik SH, Lee KY, Sohn SK, Cho CH, et al. Factors influencing survival after curative resection of pulmonary metastasis from colorectal cancer. J Korean Surg Soc 2006;70: 188-93.

6. Blalock A. Recent advances in surgery. N Engl J Med 1944;231: 261-7.

7. Ike H, Shimada H, Ohki S, Togo S, Yamaguchi S, Ichikawa Y. Results of aggressive resection of lung metastases from colorectal carcinoma detected by intensive follow-up. Dis Colon Rectum 2002;45:468-73.

8. Park IJ, Kim HC, Lee GH, Yu CS, Kim TW, Chang HM, et al. Pulmonary metastases after curative resection in patients with colorectal carcinomas. J Korean Soc Coloproctol 2003;19:307-13.

9. Sakamoto T, Tsubota N, Iwanaga K, Yuki T, Matsuoka H, Yoshimura M. Pulmonary resection for metastases from colorectal cancer. Chest 2001;119:1069-72.

10. Park JW, Moon SM, Hwang DY. The role of preoperative chest CT in the evaluation of a colorectal adenocarcinoma. J Korean Soc Coloproctol 2008;24:34-8.

11. Bhattacharjya S, Aggarwal R, Davidson BR. Intensive follow-up after liver resection for colorectal liver metastases: results of combined serial tumour marker estimations and computed tomography of the chest and abdomen: a prospective study. $\mathrm{Br} \mathrm{J}$ Cancer 2006;95:21-6.

12. Chang AE, Schaner EG, Conkle DM, Flye MW, Doppman JL, Rosenberg SA. Evaluation of computed tomography in the detection of pulmonary metastases: a prospective study. Cancer 1979;43:913-6. 
13. McIntosh J, Sylvester PA, Virjee J, Callaway M, Thomas MG. Pulmonary staging in colorectal cancer--is computerised tomography the answer? Ann R Coll Surg Engl 2005;87:331-3.

14. Povoski SP, Fong Y, Sgouros SC, Kemeny NE, Downey RJ, Blumgart LH. Role of chest CT in patients with negative chest $\mathrm{X}$ rays referred for hepatic colorectal metastases. Ann Surg Oncol 1998;5:9-15.

15. Windschitl H, Scott M, Schutt A, McCormack G, Everson L, Cullinan S, et al. Randomized phase II studies in advanced colo- rectal carcinoma: a North Central Cancer Treatment Group study. Cancer Treat Rep 1983;67:1001-8.

16. Thomford NR, Woolner LB, Clagett OT. The surgical treatment of metastatic tumors in the lungs. J Thorac Cardiovasc Surg 1965; 49:357-63.

17. Martini N, Huvos AG, Mike V, Marcove RC, Beattie EJ Jr. Multiple pulmonary resections in the treatment of osteogenic sarcoma. Ann Thorac Surg 1971;12:271-80. 